Sādhanā Vol. 40, Part 2, April 2015, pp. 425-436. (C) Indian Academy of Sciences

\title{
Closed form of optimal current waveform for class-F PA up to fourth harmonic
}

\author{
ANAMARIJA JUHAS and LADISLAV A NOVAK* \\ Faculty of Technical Sciences, Department of Power, Electronics and Communication \\ Engineering, University of Novi Sad, Trg D. Obradovica 6, 21000 Novi Sad, Serbia \\ e-mail: ladislav@uns.ac.rs
}

MS received 13 June 2012; revised 24 November 2014; accepted 25 December 2014

\begin{abstract}
In this paper, rigorous analytical derivation of the coefficients of optimal current waveform for class-F power amplifier (PA) up to fourth harmonic (dc, 1st, 2nd and 4th harmonic) is presented. The coefficients of the optimal current waveform along with related maximum attainable efficiency are provided in closed form. The results obtained are also of interest for the inverse class-F, in the case when optimal voltage waveform consists of dc, 1st, 2nd and 4th harmonic.
\end{abstract}

Keywords. Optimal waveforms; class-F PA; inverse class-F PA.

\section{Introduction}

Shaping waveforms, in order to improve efficiency, play an important role in the PA analysis. Waveforms that ensure maximum attainable efficiency are called optimal waveforms. In class-F PA and its dual, usually referred as inverse class-F PA, current and voltage waveforms can be shaped independently. In this context, optimal waveform (either current or voltage) is simply non-negative waveform with maximum possible amplitude of the first harmonic (see e.g. Raab 2001a). Common assumption for class-F is that current is non-negative waveform containing fundamental harmonic and a number of consecutive even harmonics, whereas voltage is non-negative waveform containing fundamental harmonic and a number of consecutive odd harmonics (see e.g. Raab 2001b). For inverse class-F harmonic content is dual, that is, beside fundamental harmonic voltage waveform contains even harmonics, whereas current waveform contains odd harmonics.

The problem of finding closed form expressions for non-negative waveforms with finite number of consecutive harmonics has been comprehensively considered by Fejer in his seminal paper (Fejer 1916). In the same paper, the problem of finding maximum possible amplitude of the first harmonic of cosine polynomials with consecutive harmonics has been also considered. This Fejer's result, which originally involves consecutive harmonics, is modified here to the case when third harmonic is absent. The Fejer's approach has proved to be relevant for the class-C PA as well (Juhas \& Novak 2009).

*For correspondence 
In this paper, the coefficients of the optimal current waveform, for class-F PA up to fourth harmonic, are derived in closed form. A numerical treatment of the same problem has been considered by Raab (2001a).

Class-F PA and its dual inverse class-F PA are vibrant areas of continuous interest within PA researchers and designers (see e.g. Raab 2001a; Wren \& Brazil 2005; Woo et al 2006; Schmelzer \& Long 2007; Abe et al 2008, Kim et al 2011; Tasker \& Benedikt 2011; Carrubba et al 2011, 2012; Moon et al 2012; Chen \& Peroulis 2012; Juhas \& Novak 2013).

The problem of finding optimal waveforms for PA is a part of wider problem of finding nonnegative waveforms that touch but not cross zero which has proved to be of particular practical interest (see e.g. Raab 2001b; Rhodes 2003; Cripps et al 2009; Cripps 2010; Roberg \& Popovic 2011; Juhas \& Novak 2014). Both problems are embedded in a main stream of so-called waveform engineering. As it has been observed in (Tasker \& Benedikt 2011) capturing current and voltage waveforms provides a number of advantages in the process of PA design and bring it on a level which is less dependent on device technologies. Also it was pointed out in (Tasker 2009): "Systematic experimental PA design investigation can now become waveform engineering driven; the design goal finally becoming the realization of the theoretically derived optimum waveforms." In the same paper it is stressed that "There is now a real opportunity to complement the present RF PA design approaches with waveform theory and experimental waveform information, finally allowing for robust linking of measured performance mathematically back to theoretical expectations".

In this paper we consider the problem of finding optimal waveform with dc, 1st, 2nd and 4th harmonic, that is, the problem of finding non-negative waveform

$$
i(\tau)=I_{\mathrm{dc}}\left(1+A_{1} \cos \tau+A_{2} \cos 2 \tau+A_{4} \cos 4 \tau\right) \geq 0,
$$

with maximum possible value of $A_{1}$, where $A_{4} \neq 0, \quad I_{\mathrm{dc}}>0$ and $\tau$ stands for $\omega t$.

In Section 2 we prove that optimal waveform is unique with the following set of coefficients in closed form: $A_{1}=3 / 2, \quad A_{2}=7 / 12$ and $A_{4}=-1 / 12$. An independent numerical verification of the values of these coefficients based on an optimization procedure is presented in Section 3 . In the same Section we also refer to Raab (2001a) for another independent numerical verification of our results. In Section 4 we provide closed form expressions for the maximum attainable efficiency for various versions of the class-F and inverse class-F PA, using the results obtained in Section 2.

\section{Derivation of the coefficients of the optimal current waveform}

In this Section we provide closed form expressions for coefficients of the optimal waveform of type (1), using procedure based on Fejer (1916). The Fejer's result, related to the problem of finding maximum possible amplitude of the first harmonic of cosine polynomials with consecutive harmonics, has been modified here to the case when third harmonic is absent.

Accordingly, the waveform of type (1) is non-negative if there exists a 5-tuple $\left(x_{0}, x_{1}, \ldots, x_{4}\right)$ of real numbers such that the following relations hold:

$$
\begin{gathered}
0=1-x_{0}^{2}-x_{1}^{2}-x_{2}^{2}-x_{3}^{2}-x_{4}^{2}, \\
A_{1}=2\left(x_{0} x_{1}+x_{1} x_{2}+x_{2} x_{3}+x_{3} x_{4}\right), \\
A_{2}=2\left(x_{0} x_{2}+x_{1} x_{3}+x_{2} x_{4}\right),
\end{gathered}
$$




$$
\begin{gathered}
0=2\left(x_{0} x_{3}+x_{1} x_{4}\right), \\
A_{4}=2 x_{0} x_{4} .
\end{gathered}
$$

Since $A_{4} \neq 0$ it follows that $x_{0} x_{4} \neq 0$ (see (6)). The conditions (2) and (5) impose constraints on the possible choice of elements of 5 -tuple $\left(x_{0}, x_{1}, \ldots, x_{4}\right)$.

The above problem of identifying non-negative waveform of type (1) with maximum possible amplitude of the first harmonic can be converted into the equivalent problem of finding the maximum of the function

$$
\gamma_{I}=A_{1}=2\left(x_{0} x_{1}+x_{1} x_{2}+x_{2} x_{3}+x_{3} x_{4}\right),
$$

subject to the constraints

$$
\begin{gathered}
g_{1}=1-x_{0}^{2}-x_{1}^{2}-x_{2}^{2}-x_{3}^{2}-x_{4}^{2}=0, \\
g_{2}=2\left(x_{0} x_{3}+x_{1} x_{4}\right)=0 .
\end{gathered}
$$

The reformulated problem can be reduced to the unconstrained problem of finding the maximum of the smooth function

$$
\Lambda=\gamma_{I}+\lambda_{1} g_{1}+\lambda_{2} g_{2}
$$

using Lagrange multipliers method. The following system:

$$
\begin{gathered}
\partial \Lambda / \partial x_{0}=2\left(\lambda_{1} x_{0}+x_{1}+\lambda_{2} x_{3}\right)=0 \\
\partial \Lambda / \partial x_{1}=2\left(x_{0}+\lambda_{1} x_{1}+x_{2}+\lambda_{2} x_{4}\right)=0, \\
\partial \Lambda / \partial x_{2}=2\left(x_{1}+\lambda_{1} x_{2}+x_{3}\right)=0, \\
\partial \Lambda / \partial x_{3}=2\left(\lambda_{2} x_{0}+x_{2}+\lambda_{1} x_{3}+x_{4}\right)=0, \\
\partial \Lambda / \partial x_{4}=2\left(\lambda_{2} x_{1}+x_{3}+\lambda_{1} x_{4}\right)=0,
\end{gathered}
$$

along with Eqs. (8) and (9) provide the critical points of $\Lambda$.

Let us consider linear combination of relations (11)-(15), where the coefficients of linear combination are $x_{0}, \ldots, x_{4}$, respectively. This linear combination along with (7)-(9) yields

$$
\gamma_{I}=A_{1}=-\lambda_{1}
$$

Solving (11)-(15) for $\lambda_{1}$ and $\lambda_{2}$, providing that (8)-(9) hold, yields $\lambda_{1}= \pm 3 / 2$ and $\lambda_{2}=$ $-1 / 2$. From (16) and assumption that $A_{1}>0$ it follows that $A_{1}=-\lambda_{1}=3 / 2$ and therefore

$$
\gamma_{I}=A_{1}=3 / 2 \text {. }
$$

Furthermore, solving (11), (13) and (15) for $x_{0}, \quad x_{2}, \quad x_{4}$, and substituting $\lambda_{1}=-3 / 2$ and $\lambda_{2}=-1 / 2$ into resulting relations provide $x_{0}, \quad x_{2}$ and $x_{4}$ as functions of $x_{1}$ and $x_{3}$. Further substitution of $x_{0}, \quad x_{2}$ and $x_{4}$ into (2), (4) and (6) leads to

$$
A_{2}=7 / 12, \quad A_{4}=-1 / 12 .
$$

Details of derivation of (17) and (18) are fully presented in Appendix A. 
Therefore, the optimal current waveform reads

$$
i(\tau)=I_{\mathrm{dc}}\left[1+\frac{3}{2} \cos \tau+\frac{7}{12} \cos 2 \tau-\frac{1}{12} \cos 4 \tau\right] .
$$

Using identities $\cos 2 \tau=2 \cos ^{2} \tau-1$ and $\cos 4 \tau=8 \cos ^{4} \tau-8 \cos ^{2} \tau+1$ waveform (19) can be expressed in the following factored form

$$
i(\tau)=\frac{1}{6} I_{\mathrm{dc}}(1+2 \cos \tau)^{2}(1+\cos \tau)(2-\cos \tau) .
$$

From (20) it is obvious that optimal current waveform possesses three real zeros: $\pm 2 \pi / 3$ and $\pi$. Since waveform (20) is non-negative, these zeros represent three equal global minima.

It is easy to show that $i_{\max }=i(0)=3 I_{\mathrm{dc}}$. Consequently, basic waveform parameter $\delta_{I}=i_{\max } / I_{\mathrm{dc}}$ is equal to

$$
\delta_{I}=3 .
$$

\section{Verification of the theoretical result}

Here we provide an independent verification of the results obtained in Section 2 via optimization procedure treated numerically. This approach, which is different from that of Section 2, involves process of finding maximum of a minimum function. The values obtained for the coefficients of the optimal waveform, through this optimization procedure, are in full agreement with the values of the coefficients obtained in closed form in Section 2.

To show that, notice that waveform of type (1) can be expressed in the following form

$$
i(\tau) / I_{\mathrm{dc}}=1+A_{1}\left(\cos \tau+b_{2} \cos 2 \tau+b_{4} \cos 4 \tau\right)=1+A_{1} f_{b_{2}, b_{4}}(\tau),
$$

where

$$
A_{2}=A_{1} b_{2}, \quad A_{4}=A_{1} b_{4}
$$

and

$$
f_{b_{2}, b_{4}}(\tau)=\cos \tau+b_{2} \cos 2 \tau+b_{4} \cos 4 \tau .
$$

Let us now consider the problem of finding maximum possible value of coefficient $b_{1}$ that satisfy

$$
1+b_{1} f_{b_{2}, b_{4}}(\tau) \geq 0,
$$

which is equivalent to the original problem. Furthermore, let

$$
F\left(b_{2}, b_{4}\right)=\min _{\tau} f_{b_{2}, b_{4}}(\tau),
$$

be a minimum function associated with $f_{b_{2}, b_{4}}(\tau)$. According to $(24), b_{1}$ and $F\left(b_{2}, b_{4}\right)$ satisfy the relation

$$
1+b_{1} F\left(b_{2}, b_{4}\right) \geq 0 .
$$

Clearly, for any prescribed pair $\left(b_{2}, b_{4}\right)$, there is a unique maximal value of coefficient $b_{1}$, denoted by $B_{1}\left(b_{2}, b_{4}\right)$, for which (26) becomes equality. In other words, the following relation holds

$$
B_{1}\left(b_{2}, b_{4}\right)=-\frac{1}{F\left(b_{2}, b_{4}\right)}=-\frac{1}{\min _{\tau} f_{b_{2}, b_{4}}(\tau)} .
$$


Furthermore, maximal value of $B_{1}\left(b_{2}, b_{4}\right)$ taken over all pairs $\left(b_{2}, b_{4}\right)$, denoted by $B_{1}^{*}$, can be calculated from

$$
B_{1}^{*}=\max _{b_{2}, b_{4}}\left(B_{1}\left(b_{2}, b_{4}\right)\right)=-\frac{1}{\max _{b_{2}, b_{4}}\left(\min _{\tau} f_{b_{2}, b_{4}}(\tau)\right)} .
$$

Notice that $B_{1}^{*}$ is the maximum possible value of $A_{1}$ of the waveform (22).

Typically, minimum function is not differentiable (see e.g. Bogaevsky 2002). Therefore, $B_{1}^{*}$ cannot be derived by approach based on finding critical points. This fact explains why we opted for the approach presented in Section 2.

In what follows we treat the optimization problem described by (28), numerically. An algorithm for numerical calculation of coefficients of the optimal waveform is presented on the next page.

Short description of the algorithm is as follows: in steps $2-5$ we choose a grid in parameter space $\left(b_{2}, b_{4}\right)$; in steps 6-7 we choose a number of points $\tau$ on interval $\left[\tau_{\min }, \tau_{\max }\right]$; in steps 8-15 we calculate $B_{1}\left(b_{2}, b_{4}\right)$ for each pair $\left(b_{2}, b_{4}\right)$; in steps $16-18$ we find $B_{1}^{*}$ and corresponding pair $\left(b_{2}^{*}, b_{4}^{*}\right)$; and in step 19 we calculate the coefficients of the optimal waveform: $A_{1}, A_{2}$ and $A_{4}$.

It is obvious that the accuracy of the numerical values of the coefficients obtained by the algorithm depends on chosen grid in parameter space $\left(b_{2}, b_{4}\right)$ and step-size for $\tau$. For example, for $b_{2} \in[-1,1], b_{2, \text { step }}=10^{-4}, b_{4} \in[-0.5,0.5], b_{4 \text {,step }}=5 \cdot 10^{-5}, \tau \in[0, \pi]$ (due to symmetry of cosine waveforms) and $\tau_{\text {step }}=10^{-4}$ we obtain $B_{1}^{*}=1.5000, b_{2}^{*}=0.3889$ and $b_{4}^{*}=-0.0556$. Corresponding numerical values of the coefficients of the optimal waveform are $A_{1}=1.5000, A_{2}=0.5833$ and $A_{4}=-0.0833$. These values are in agreement with the closed form expressions for the coefficients obtained in Section 2 (see (17) and (18)).

We also would like to refer to the seminal paper (Raab 2001a) for another independent numerical verification of our result. The current waveform used in (Raab 2001a), after shifting by $3 \pi / 2$ to the left, can be converted into the form:

$$
i(\tau)=I_{\mathrm{dc}}\left(1+\left(I_{o m} / I_{\mathrm{dc}}\right) \cos \tau+\left(I_{2 m} / I_{\mathrm{dc}}\right) \cos 2 \tau-\left(I_{4 m} / I_{\mathrm{dc}}\right) \cos 4 \tau\right) .
$$

Comparison of (29) with (1) and usage of table II of (Raab 2001a) leads to the following relations: $A_{1}=I_{o m} / I_{\mathrm{dc}}=1.5000, A_{2}=I_{2 m} / I_{\mathrm{dc}}=0.5835$ and $A_{4}=-I_{4 m} / I_{\mathrm{dc}}=-0.0834$. These numerical results also confirm our analytically obtained results.

\section{Maximum attainable efficiency}

In this Section we provide closed form expressions for maximum attainable efficiency in the following four cases:

(i) finite harmonic class-F PA up to fifth harmonic (Subsection 4.1),

(ii) finite harmonic inverse class-F PA up to fifth harmonic (Subsection 4.1),

(iii) class-F PA with square-wave voltage waveform and current waveform up to fourth harmonic (Subsection 4.2),

(iv) inverse class-F PA with square-wave current waveform and voltage waveform up to fourth harmonic (Subsection 4.2). 


\section{Algorithm :}

Input:

$$
\begin{aligned}
& b_{2, \text { min }}, \quad b_{2, \text { max }}, i_{\text {max }} / / b_{2}\left(1 . . i_{\max }\right) \in\left[b_{2, \text { min }}, b_{2, \text { max }}\right] \\
& b_{4, \text { min }}, \quad b_{4, \text { max }}, \quad j_{\max } / / b_{4}\left(1 . . j_{\max }\right) \in\left[b_{4, \text { min }}, b_{4, \text { max }}\right] \\
& \tau_{\min }, \tau_{\max }, \quad k_{\max } / / \tau\left(1 . . k_{\max }\right) \in\left[\tau_{\min }, \tau_{\max }\right]
\end{aligned}
$$

Output:

$$
A_{1}, A_{2}, A_{4} / / \text { Coefficients of optimal waveform }
$$

\section{1. begin}

2. $b_{2, \text { step }} \leftarrow\left(b_{2, \text { max }}-b_{2, \text { min }}\right) /\left(i_{\max }-1\right)$;

3. for $i=1$ to $i_{\max }$ do; $b_{2}(i) \leftarrow b_{2, \text { min }}+(i-1) b_{2, \text { step }}$; end for

4. $\quad b_{4, \text { step }} \leftarrow\left(b_{4, \text { max }}-b_{4, \min }\right) /\left(j_{\max }-1\right)$;

5. for $j=1$ to $j_{\max }$ do; $b_{4}(j) \leftarrow b_{4, \min }+(j-1) b_{4, \text { step }}$; end for

6. $\tau_{\text {step }} \leftarrow\left(\tau_{\max }-\tau_{\min }\right) /\left(k_{\max }-1\right)$;

7. for $k=1$ to $k_{\max }$ do; $\tau(k) \leftarrow \tau_{\min }+(k-1) \tau_{\text {step }}$; end for

8. for $i=1$ to $i_{\max }$ do

9. for $j=1$ to $j_{\max }$ do

10. for $k=1$ to $k_{\max }$ do

11. $\quad f(k) \leftarrow \cos (\tau(k))+b_{2}(i) \cos (2 \tau(k))+b_{4}(j) \cos (4 \tau(k))$;

12. end for // end $k$-loop

13. $F_{\min } \leftarrow \min _{k} f\left(1 . . k_{\max }\right) ; \quad B_{1}(j, i) \leftarrow-1 / F_{\text {min }}$;

14. end for // end $j$-loop

15. end for // end $i-$ loop

16. $B_{1}^{*} \leftarrow \max _{i, j}\left(B_{1}\left(1 . . j_{\max }, 1 . . i_{\max }\right)\right)$;

17. find $\left(j^{*}, i^{*}\right)$ such that $B_{1}\left(j^{*}, i^{*}\right)=B_{1}^{*}$;

18. $b_{2}^{*} \leftarrow b_{2}\left(i^{*}\right) ; b_{4}^{*} \leftarrow b_{4}\left(j^{*}\right)$;

19. $A_{1} \leftarrow B_{1}^{*} ; A_{2} \leftarrow B_{1}^{*} b_{2}^{*} ; A_{4} \leftarrow B_{1}^{*} b_{4}^{*}$;

20. end. 
In class-F (inverse class-F) PA with $N$ harmonics the voltage (current) waveform contains dc component and all odd harmonics up to $N$, whereas the current (voltage) waveform contains dc component, fundamental and all even harmonics up to $N$.

The efficiency and power-output capability of PA can be expressed via "basic waveform parameters" $\gamma_{I}, \gamma_{V}, \delta_{I}$ and $\delta_{V}$ (see e.g. Raab 2001a). For current waveform

$$
i(\tau)=I_{\mathrm{dc}}\left(1+A_{1} \cos \tau+\sum_{m=2}^{M} A_{m} \cos m \tau\right) \geq 0,
$$

the parameters $\gamma_{I}$ and $\delta_{I}$ are related to $A_{1}, I_{\mathrm{dc}}$ and $i_{\max }=\max (i(\tau))$ as follows:

$$
\gamma_{I}=A_{1}, \quad \delta_{I}=i_{\max } / I_{\mathrm{dc}} .
$$

Similarly, for the voltage waveform

$$
v(\tau)=V_{\mathrm{dc}}\left(1-B_{1} \cos \tau-\sum_{k=2}^{K} B_{k} \cos k \tau\right) \geq 0,
$$

the parameters $\gamma_{V}$ and $\delta_{V}$ are related to $B_{1}, V_{\mathrm{dc}}$ and $v_{\max }=\max (v(\tau))$ as follows:

$$
\gamma_{V}=B_{1}, \quad \delta_{V}=v_{\max } / V_{\mathrm{dc}}
$$

The efficiency of PA can be expressed via $\gamma_{I}$ and $\gamma_{V}$ waveform parameters (see e.g. Raab 2001a):

$$
\eta=\frac{\gamma_{I} \gamma_{V}}{2}
$$

The power-output capability is equal to (see e.g. Raab 2001a):

$$
P_{\max }=\frac{\gamma_{I} \gamma_{V}}{2 \delta_{I} \delta_{V}}=\frac{\eta}{\delta_{I} \delta_{V}}
$$

\subsection{Finite harmonic class-F and inverse class-F PA operation up to fifth harmonic}

In this Subsection we provide closed form expressions for maximum attainable efficiency of finite harmonic class-F PA operation up to fifth harmonic. We would like to point out here that closed form expressions for maximum attainable efficiency of finite harmonic class-F PA operation up to third harmonic have been provided by Raab in (Raab 2001a). Concerning cases with four and five harmonics, in the same paper, maximum attainable efficiencies have been calculated numerically.

In what follows we provide closed form expressions for maximum attainable efficiency of finite harmonic class-F and inverse class-F PA with four and five harmonics.

For finite harmonic class-F PA with $N=4$ and $N=5$ harmonics, basic waveform parameters of optimal current waveform are $\gamma_{I}=3 / 2$ and $\delta_{I}=3$ (this paper, see (17) and (21)). Basic waveform parameters of optimal voltage waveform for $N=4$ are equal to $\gamma_{V}=2 / \sqrt{3}$ and $\delta_{V}=2$ (see e.g. Raab 2001a). For $N=5$ basic waveform parameters of optimal voltage waveform are equal to $\gamma_{V}=(1+\sqrt{2}) / 2$ (Rhodes 2003) and $\delta_{V}=2$ (since it contains only odd harmonics). 
Table 1. Maximum attainable efficiency and corresponding power-output capability of finite harmonic class-F PA up to fifth harmonic.

\begin{tabular}{ccccccccc}
\hline & \multicolumn{2}{c}{ Harmonics } & & & & & \\
\cline { 2 - 4 }$N$ & $i(\tau)$ & $v(\tau)$ & $\gamma_{I}$ & $\gamma_{V}$ & & $\delta_{I}$ & $\delta_{V}$ & $P_{\max }$ \\
\hline 1 & 1 & 1 & 1 & 1 & $1 / 2=0.5000$ & 2 & 2 & $1 / 8=0.1250$ \\
2 & 1,2 & 1 & $\sqrt{2}$ & 1 & $\sqrt{2} / 2=0.7071$ & $\sqrt{2}+3 / 2$ & 2 & $3 / \sqrt{2}-2=0.1213$ \\
3 & 1,2 & 1,3 & $\sqrt{2}$ & $2 / \sqrt{3}$ & $\sqrt{2 / 3}=0.8165$ & $\sqrt{2}+3 / 2$ & 2 & $\sqrt{6}-4 / \sqrt{3}=0.1401$ \\
4 & $1,2,4$ & 1,3 & $3 / 2$ & $2 / \sqrt{3}$ & $\sqrt{3} / 2=0.8660$ & 3 & 2 & $\sqrt{3} / 12=0.1443$ \\
5 & $1,2,4$ & $1,3,5$ & $3 / 2$ & $(1+\sqrt{2}) / 2$ & $3(1+\sqrt{2}) / 8=0.9053$ & 3 & 2 & $(1+\sqrt{2}) / 16=0.1509$ \\
\hline
\end{tabular}

The analytical expressions for maximum attainable efficiency and corresponding poweroutput capability of finite harmonic class-F PA operation up to fifth harmonic are given in table 1.

Optimal current and voltage waveforms of finite harmonic class-F PA operation with four and five harmonics are presented in figure 1 .

Maximum attainable efficiency and corresponding power-output capability of finite harmonic inverse class-F PA operation up to fifth harmonic (table 2) can be obtained directly from efficiency and power-output capability of finite harmonic class-F, by interchanging the parameters of voltage and current waveforms. Therefore, the maximum attainable efficiency and corresponding power-output capability are the same for finite harmonic class-F and finite harmonic inverse class-F PA operation, with the same number of harmonics.

\subsection{Class-F and inverse class-F PA operation}

Basic waveform parameters of square-wave voltage waveform are $\gamma_{V}=4 / \pi$ and $\delta_{V}=2$ (see e.g. Raab 2001a). Basic parameters of the optimal current waveform are $\gamma_{I}=\sqrt{2}$ and $\delta_{I}=$ $\sqrt{2}+3 / 2$ when it contains dc component, fundamental and second harmonic $(M=2)$ (see e.g. Raab 2001a), or $\gamma_{I}=3 / 2$ and $\delta_{I}=3$ when it contains dc component, fundamental, second and fourth harmonic $(M=4)$ (this paper, see (17) and (21)). Maximum attainable efficiency and corresponding power-output capability of class-F PA operation with square-wave voltage waveform and current waveform up to fourth harmonic are given in table 3.

Maximum attainable efficiency and corresponding power-output capability of inverse class-F PA operation with square-wave current waveform and voltage waveform up to fourth harmonic (table 4) can be obtained directly from previous case by interchanging the parameters of voltage and current waveforms.
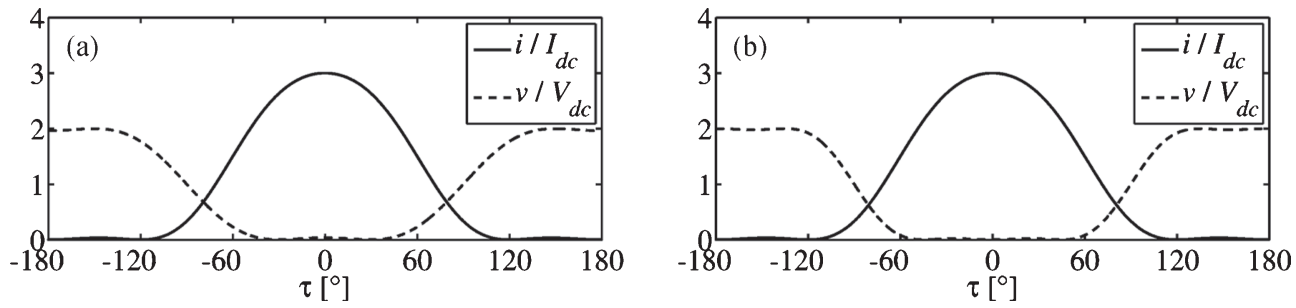

Figure 1. Optimal current (solid line) and voltage (dashed line) waveforms of finite harmonic class-F PA for (a) $N=4$ and (b) $N=5$. 
Table 2. Maximum attainable efficiency and corresponding power-output capability of finite harmonic inverse class-F PA up to fifth harmonic.

\begin{tabular}{ccccccccc}
\hline & \multicolumn{2}{c}{ Harmonics } & & & & & \\
\cline { 2 - 4 } & $i(\tau)$ & $v(\tau)$ & $\gamma_{I}$ & $\gamma_{V}$ & $\eta$ & $\delta_{I}$ & $\delta_{V}$ & $P_{\max }$ \\
\hline 1 & 1 & 1 & 1 & 1 & $1 / 2=0.5000$ & 2 & 2 & $1 / 8=0.1250$ \\
2 & 1 & 1,2 & 1 & $\sqrt{2}$ & $\sqrt{2} / 2=0.7071$ & 2 & $\sqrt{2}+3 / 2$ & $3 / \sqrt{2}-2=0.1213$ \\
3 & 1,3 & 1,2 & $2 / \sqrt{3}$ & $\sqrt{2}$ & $\sqrt{2 / 3}=0.8165$ & 2 & $\sqrt{2}+3 / 2$ & $\sqrt{6}-4 / \sqrt{3}=0.1401$ \\
4 & 1,3 & $1,2,4$ & $2 / \sqrt{3}$ & $3 / 2$ & $\sqrt{3} / 2=0.8660$ & 2 & 3 & $\sqrt{3} / 12=0.1443$ \\
5 & $1,3,5$ & $1,2,4$ & $(1+\sqrt{2}) / 2$ & $3 / 2$ & $3(1+\sqrt{2}) / 8=0.9053$ & 2 & 3 & $(1+\sqrt{2}) / 16=0.1509$ \\
\hline
\end{tabular}

Table 3. Maximum attainable efficiency and corresponding power-output capability of class-F PA with square-wave voltage waveform and current waveform up to fourth harmonic.

\begin{tabular}{|c|c|c|c|c|c|c|c|}
\hline$M$ & $\begin{array}{l}\text { Harmonics } \\
\text { in } i(\tau)\end{array}$ & $\gamma_{I}$ & $\gamma_{V}$ & $\eta$ & $\delta_{I}$ & $\delta_{V}$ & $P_{\max }$ \\
\hline 1 & 1 & 1 & $4 / \pi$ & $2 / \pi=0.6366$ & 2 & 2 & $1 /(2 \pi)=0.1592$ \\
\hline 2 & 1,2 & $\sqrt{2}$ & $4 / \pi$ & $2 \sqrt{2} / \pi=0.9003$ & $\sqrt{2}+3 / 2$ & 2 & $(6 \sqrt{2}-8) / \pi=0.1545$ \\
\hline 4 & $1,2,4$ & $3 / 2$ & $4 / \pi$ & $3 / \pi=0.9549$ & 3 & 2 & $1 /(2 \pi)=0.1592$ \\
\hline
\end{tabular}

Table 4. Maximum attainable efficiency and corresponding power-output capability of inverse class-F PA with square-wave current waveform and voltage waveform up to fourth harmonic.

\begin{tabular}{|c|c|c|c|c|c|c|c|}
\hline$K$ & $\begin{array}{l}\text { Harmonics } \\
\quad \text { in } v(\tau)\end{array}$ & $\gamma_{I}$ & $\gamma_{V}$ & $\eta$ & $\delta_{I}$ & $\delta_{V}$ & $P_{\max }$ \\
\hline 1 & 1 & $4 / \pi$ & 1 & $2 / \pi=0.6366$ & 2 & 2 & $1 /(2 \pi)=0.1592$ \\
\hline 2 & 1,2 & $4 / \pi$ & $\sqrt{2}$ & $2 \sqrt{2} / \pi=0.9003$ & 2 & $\sqrt{2}+3 / 2$ & $(6 \sqrt{2}-8) / \pi=0.1545$ \\
\hline 4 & $1,2,4$ & $4 / \pi$ & $3 / 2$ & $3 / \pi=0.9549$ & 2 & 3 & $1 /(2 \pi)=0.1592$ \\
\hline
\end{tabular}

\section{Conclusions}

In this paper, the coefficients of optimal current waveform up to fourth harmonic, providing that third harmonic is absent, are derived in closed form. The optimal current waveform reads

$$
i(\tau)=I_{\mathrm{dc}}\left[1+\frac{3}{2} \cos \tau+\frac{7}{12} \cos 2 \tau-\frac{1}{12} \cos 4 \tau\right] .
$$

The closed form expressions for basic waveform parameters of this waveform are $\gamma_{I}=3 / 2$ and $\delta_{I}=3$. Derivation presented in Section 2 is also applicable to optimal waveform containing fundamental, second and fourth harmonic irrespective of the physical nature. Therefore the same expression, up to physical nature of multiplicative constant, can be used to describe optimal voltage waveform containing fundamental, second and fourth harmonic.

Basic waveform parameters of optimal waveform with fundamental, second and fourth harmonic (this paper), along with well-known basic waveform parameters of optimal waveforms containing fundamental and second harmonic (Raab 2001a), fundamental and third harmonic (Raab 2001a), or fundamental, third and fifth harmonic (Rhodes 2003), enable us to provide closed form expressions for maximum attainable efficiencies and corresponding power output capabilities in the following four cases: (i) finite harmonic class-F PA up to fifth harmonic 
(table 1), (ii) finite harmonic inverse class-F PA up to fifth harmonic (table 2), (iii) class-F PA with square-wave voltage waveform and current waveform up to fourth harmonic (table 3 ) and (iv) inverse class-F PA with square-wave current waveform and voltage waveform up to fourth harmonic (table 4).

\section{Acknowledgements}

This work is supported by Serbian Ministry of Education, Science and Technology Development as a part of the Project TP32016.

\section{Appendix A}

Full account of details of derivation of (17) and (18) (Section 2) is provided in this appendix.

From (11), (13) and (15) we obtain

$$
x_{0}=-\frac{x_{1}+\lambda_{2} x_{3}}{\lambda_{1}}, \quad x_{2}=-\frac{x_{1}+x_{3}}{\lambda_{1}}, \quad x_{4}=-\frac{\lambda_{2} x_{1}+x_{3}}{\lambda_{1}} .
$$

Combining (A.1) and (12) yields

$$
x_{1}\left(2-\lambda_{1}^{2}+\lambda_{2}^{2}\right)+x_{3}\left(1+2 \lambda_{2}\right)=0,
$$

whereas combining (A.1) and (14) yields

$$
x_{3}\left(2-\lambda_{1}^{2}+\lambda_{2}^{2}\right)+x_{1}\left(1+2 \lambda_{2}\right)=0 .
$$

According to (A.1), $x_{1}$ and $x_{3}$ are not simultaneously equal to zero, since otherwise, it would lead to $x_{0}=x_{2}=x_{4}=0$, which together with $x_{1}=x_{3}=0$ contradicts (8).

Equations (A.2) and (A.3) can be considered as a system of linear equations in terms of (2 $\left.\lambda_{1}^{2}+\lambda_{2}^{2}\right)$ and $\left(1+2 \lambda_{2}\right)$. Thus we have the following two cases: either (i) $x_{1}^{2}-x_{3}^{2}=0$ or (ii) $x_{1}^{2}-x_{3}^{2} \neq 0$. In what follows we show that case (i) leads to contradiction, while case (ii) leads to the optimal waveform given by (19).

(i) From $x_{1}= \pm x_{3}$, taking into account that at least one of $x_{1}$ and $x_{3}$ is not equal to zero, it follows that $x_{1} \neq 0$ and $x_{3} \neq 0$. Substitution of $x_{1}= \pm x_{3}$ into (A.1) leads to $x_{0}= \pm x_{4}$. Furthermore, inserting $x_{1}= \pm x_{3}$ and $x_{0}= \pm x_{4}$ into (9) implies $x_{0}=x_{4}=0$, since $x_{1} \neq 0$. This contradict our assumption that $A_{4}=2 x_{0} x_{4} \neq 0$.

(ii) The determinant of the system (A.2)-(A.3) is non-zero and consequently it has trivial solution only: $2-\lambda_{1}^{2}+\lambda_{2}^{2}=0$ and $1+2 \lambda_{2}=0$, that is,

$$
\lambda_{2}=-1 / 2, \quad \lambda_{1}^{2}=9 / 4
$$

Since $A_{1}>0$, from (16) and (A.4) it follows that (17) holds. Substituting $\lambda_{1}=-A_{1}=-3 / 2$ and $\lambda_{2}=-1 / 2$ into (A.1) we obtain

$$
x_{0}=\frac{2 x_{1}-x_{3}}{3}, \quad x_{2}=\frac{2 x_{1}+2 x_{3}}{3}, \quad x_{4}=\frac{-x_{1}+2 x_{3}}{3} .
$$


From (2), using (A.5), it follows that

$$
2 x_{1}^{2}+2 x_{3}^{2}=1
$$

From (A.5), (A.6) and (5) it follows that

$$
8 x_{1} x_{3}=1
$$

Finally, substituting (A.5)-(A.7) into (4) and (6) leads to (18).

\section{References}

Abe Y, Ishikawa R and Honjo K 2008 Inverse class-F AlGaN/GaN HEMT microwave amplifier based on lumped element circuit synthesis method. IEEE Trans. Microw. Theory Tech. 56: 2748-2753

Bogaevsky I A 2002 Perestroikas of shocks and singularities of the minimum functions. Physica D 173: $1-28$

Carrubba V, Clarke A L, Akmal M, Lees J, Benedikt J, Tasker P J and Cripps S C 2011 On the extension of the continuous class-F mode power amplifier. IEEE Trans. Microw. Theory Tech. 59: 1294-1303

Carrubba V, Akmal M, Quay R, Lees J, Benedikt J, Cripps S C and Tasker P J 2012 The continuous inverse class-F mode with resistive second-harmonic impedance. IEEE Trans. Microw. Theory Tech. 60: 19281936

Chen K and Peroulis D 2012 Design of broadband highly efficient harmonic-tuned power amplifier using in-band continuous class- $\mathrm{F}^{-1} / \mathrm{F}$ mode transferring. IEEE Trans. Microw. Theory Tech. 60: 4107-4116

Cripps S C 2010 Grazing zero [microwave bytes]. IEEE Microw. Mag. 11(7): 24-34

Cripps S C, Tasker P J, Clarke A L, Lees J and Benedikt J 2009 On the continuity of high efficiency modes in linear RF power amplifiers. IEEE Microw. Wireless Compon. Lett. 19: 665-667

Fejer L 1916 Über trigonometrische polynome. (in German). J. Reine Angew. Math. 146: 53-82

Juhas A and Novak L A 2009 Comments on "Class-E, class-C, and class-F power amplifier based upon a finite number of harmonics". IEEE Trans. Microw. Theory Tech. 57: 1623-1625

Juhas A and Novak L A 2013 Maximally flat waveforms with finite number of harmonics in class-F power amplifiers. Math. Probl. Eng. 2013: Article ID 169590, 9 pages

Juhas A and Novak L A 2014 General description of nonnegative waveforms up to second harmonic for power amplifier modelling. Math. Probl. Eng. 2014: Article ID 709762, 18 pages

Kim J H, Jo G D, Oh J H, Kim Y H, Lee K C and Jung J H 2011 Modeling and design methodology of high-efficiency class-F and class- $\mathrm{F}^{-1}$ power amplifiers. IEEE Trans. Microw. Theory Tech. 59: 153-165

Moon J, Jee S, Kim J, Kim J and Kim B 2012 Behaviors of class-F and class- $\mathrm{F}^{-1}$ amplifiers. IEEE Trans. Microw. Theory Tech. 60: 1937-1951

Raab F H 2001 a Maximum efficiency and output of class-F power amplifiers. IEEE Trans. Microw. Theory Tech. 49: 1162-1165

Raab F H 2001b Class-E, class-C, and class-F power amplifiers based upon a finite number of harmonics. IEEE Trans. Microw. Theory Tech. 49: 1462-1468

Rhodes J D 2003 Output universality in maximum efficiency linear power amplifiers. Int. J. Circuit Theory Appl. 31: 385-405

Roberg M and Popovic Z 2011 Analysis of high-efficiency power amplifiers with arbitrary output harmonic terminations. IEEE Trans. Microw. Theory Tech. 59: 2037-2048

Schmelzer D and Long S I 2007 A GaN HEMT class F amplifier at 2GHz with 80\% PAE. IEEE J. SolidState Circuits 42: 2130-2136

Tasker P J 2009 Practical waveform engineering. IEEE Microw. Mag. 10(7): 65-76

Tasker P J and Benedikt J 2011 Waveform inspired models and the harmonic balance emulator. IEEE Microw. Mag. 12(2): 38-54 
Woo Y Y, Yang Y and Kim B 2006 Analysis and experiments for high-efficiency class-F and inverse class-F power amplifiers. IEEE Trans. Microw. Theory Tech. 54: 1969-1974

Wren M and Brazil T J 2005 Experimental class-F power amplifier design using computationally efficient and accurate large-signal pHEMT model. IEEE Trans. Microw. Theory Tech. 53: 1723-1731 